\title{
EJERCICIOS ADECUADOS DE RESISTENCIA ABDOMINAL: UNA HERRAMIENTA PARA LA PREVENCIÓN CONTRA LUMBALGIAS
}

Carlos Álvarez Bogantes

Escuela Ciencias del Deporte,

Facultad de Ciencias de la Salud,

Universidad Nacional

calvarez@una.ac.cr

RESUMEN

El propósito de este artículo fue el de analizar la técnica tradicional de los ejercicios abdominales, con el fin de dar recomendaciones alternativas que garanticen una buena ejecución sin riesgos. Además, presenta algunas recomendaciones que ayudarán a prescribir el ejercicio abdominal para la salud.

PALABRAS CLAVES: Músculos erectores, abdominales y valsalva.

\section{APPROPRIATE ABDOMINAL STRENGTH EXERCISES: A TOOL FOR THE PREVENTION OF LUMBAGO}

\section{ABSTRACT}

The purpose of this article was to analyze the technique traditionally used in abdominal exercises in order to give alternatives that would guarantee a correct risk-free execution. In addition, some recommendations are provided that would help prescribe abdominal exercises for health purposes.

KEY WORDS: Abdominal muscles and valsalva.

\section{INTRODUCCIÓN}

¡Imposible de creer, pero era una realidad! Ahí, en la cama, yacía María, la vecina pizpireta, sin poder mover ninguno de sus preciados músculos abdominales. El solo intento de mover alguno de los músculos para provocar una sonrisa, era motivo de dolor, este estaba en su parte lumbar y era fuera de este mundo, perdón, fuera de este mundo, pero causado en este mundo. Para hacer de una larga historia algo impactante, esta amiga quiso deshacerse de sus llantas recurriendo al viejo y gastado método de realizar 100 ejercicios abdominales, con las piernas estiradas, manos detrás de la cabeza y con una compañera sosteniéndole las rodillas. El instructor de su primer y último día, es 
una persona que considera que el dolor es parte esencial a la hora de realizar actividad física o ejercicio, sin importar si la persona que lo ejecuta es un atleta o una persona sedentaria, como en el caso doña María que tenía veinte años de solo correr al refrigerador para comerse algo.

Los ejercicios abdominales han sido utilizados en el medio costarricense no solo con la idea de desarrollar resistencia muscular, pero primordialmente con la esperanza de disminuir grasa abdominal. Para desdicha de los miles de personas que los han ejecutado con ese fin, esa estrategia es poco válida y riesgosa si se ejecutan abdominales inapropiados.

Con lo anterior en mente, se pretende presentar los elementos que hacen del abdominal tradicional inapropiado, especialmente, cuando se trabaja con poblaciones sedentarias. A la vez que se analiza un tipo de abdominal conveniente para la mayoría de las personas y que garantiza un nivel de seguridad alto.

\section{DESARROLLO}

La mecánica a la hora de ejecutar los abdominales ha evolucionado en forma radical, llegándose a la práctica de ejercicios más simples y seguros para los ejecutantes. Recientemente, el abdominal causante de todos los males vividos por mi vecina, ha sido fuertemente cuestionado, especialmente cuando se trabaja con personas sedentarias. Algunos especialistas consideran que el tener mala resistencia abdominal es un elemento que contribuye a desarrollar lumbalgias o problemas lumbares (Craig and McGill, 1997), pero también lo es, el ejecutar abdominales tradicionales, ya que este ejercicio es considerado un precursor de problemas en la espalda baja, debido a que pone una carga de compresión alta en esa zona (McGill, 1992). Al ejecutar el abdominal tradicional colocando las manos detrás de la cabeza, podría causar hiperflexión del cuello y provocar así una lesión del mismo o de los discos intervertebrales, especialmente cuando se presenta la fatiga y se pone mayor presión sobre el área. Adicionalmente, si se realiza con las piernas extendidas y sosteniendo los pies, se obtiene una participación precoz de los flexores de la cadera y un aumento de la presión en la parte lumbar (ver dibujo 1.a) (Van Gender y Marks, 1987). Cuando el área lumbar pierde contacto con el suelo, los flexores de la cadera se involucran en mayor medida, disminuyendo la participación de los abdominales. La flexión de la cadera es iniciada por la contracción concéntrica del músculo iliaco, el psoas mayor, menor y el recto femoral. Estos músculos contribuyen a la flexión de la cadera y en alguna medida participan en la hiperextensión lumbar, aumentando la rotación de la pelvis en la dirección anterior y aumentando la presión intradiscal (Craig and McGill, 1997). En el caso de una persona entrenada, con músculos abdominales resistentes y fuertes, que realice el abdominal mencionado, se podría disminuir la acción de hiperextensión lumbar. Sin embargo, el caso es que la mayoría de personas carecen de esa fortaleza abdominal, por lo que el ser conservador con este abdominal es lo recomendado para evitar el riesgo de dolor o lesión lumbar.

En la búsqueda de un abdominal más seguro se ha llegado a proponer que al realizar la ejecución del tradicional se flexionen las rodillas en un ángulo de 90 grados, que las manos se coloquen lateralmente y que el soporte de los pies sea eliminado (ver dibujo 1.c). Estas modificaciones han mejorado sustancialmente la mecánica de ejecución, aunque el ejecutar este ejercicio a través de todo el rango de movimiento sigue siendo una preocupación. Esto ha llevado a agregar un elemento más de cambio, y es el de disminuir en dos tercios el rango del movimiento, lo que evita que la parte lumbar se despegue del piso, manteniendo una restricción significativa en la participación de los flexores de la cadera (dibujo 2) (Juker, McGill and Kropf, 1996). Una recomendación adicional que es de gran validez, es que durante la ejecución de este ejercicio hay que evitar mantener la respiración, lo cuál se le llama la maniobra de valsalva, ya que al mantener la respiración aumenta la presión intratorácica, restringiendo el flujo normal de sangre al área, pudiendo obtenerse cambios 
bruscos en la presión arterial. Esta situación es de gran importancia, especialmente cuando se trabaja con sujetos de alto riesgo (Lamb, 1994).

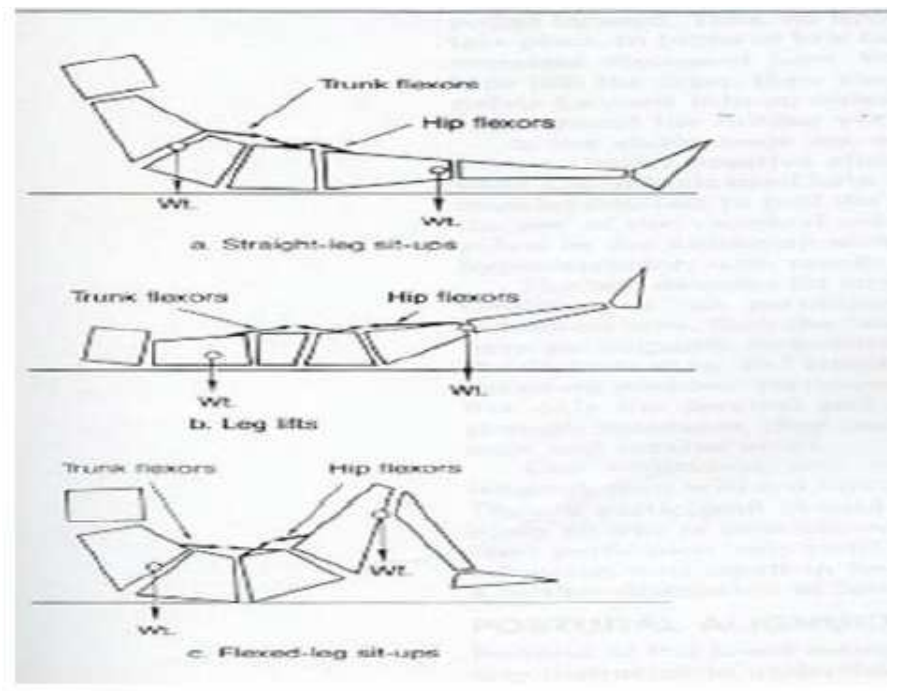

Fuente: Van Gender y Marks (1987)

Es de suma importancia que la resistencia muscular abdominal, que es un componente de la Aptitud Física para la Salud, sea evaluada por todo profesional del área antes de que cualquier persona inicie un plan de actividad física. Con esto nos garantizamos no sólo que la persona aprenda la forma correcta de ejecutar la actividad y de que entienda que esencialmente esta actividad es realizada para disminuir riesgos a nivel lumbar, pero también nos ofrece la posibilidad de prescribirle a la persona el ejercicio en forma apropiada y segura.

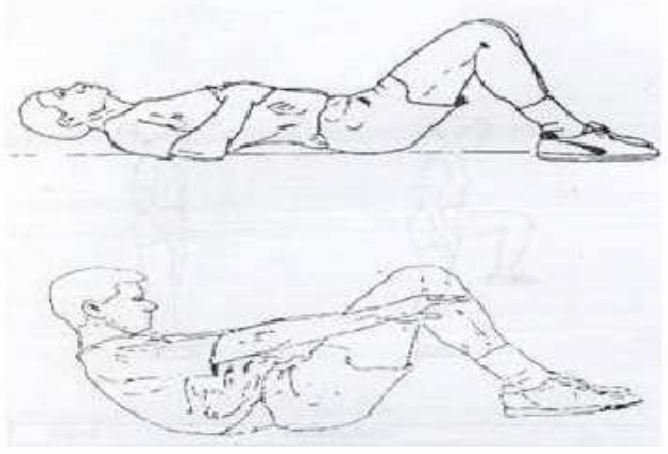

Fuente: Alvarez (1997)

Las siguientes son las indicaciones apropiadas para que se ejecute el abdominal modificado. Además, se presentan las normas obtenidas con una población mixta de dos mil personas, con edades de 25 a 50 años, que fueron atendidos en el Instituto de Medicina Deportiva de la Universidad Nacional durante los años de 1994 y 1998. 
Tabla 1. Normas abdominal modificado

\begin{tabular}{ccc}
\hline Nivel de aptitud & Hombres & Mujeres \\
\hline Excelente & +59 & +45 \\
Bueno & $50-58$ & $39-44$ \\
Regular & $39-49$ & $30-38$ \\
Bajo & -38 & -29 \\
\hline
\end{tabular}

Una vez que la persona ha sido evaluada y clasificada, usando las normas anteriores, corresponde realizar una prescripción del ejercicio, la cuál podemos efectuar aplicando las indicaciones del cuadro siguiente.

Consideren el ejemplo de una mujer, que al ejecutar la prueba del abdominal modificado, realizó 25 abdominales. Si se busca este resultado en las normas presentadas anteriormente, se darán cuenta que esta mujer tiene una resistencia abdominal baja, por lo que se deberá prescribir el ejercicio con mayor prudencia. La prescripción hipotética de nuestro caso sería:

Tabla 2. Entrenamiento de resistencia abdominal para la salud

\begin{tabular}{cc}
\hline Modalidad & Abdominal modificado \\
\hline Series & 1 serie mínima \\
Intensidad & $50 \%$ de lo máximo \\
Progresión & De un 5 a un $10 \%$ de aumento por mes \\
Frecuencia & 2 ó 3 veces por semana \\
\hline
\end{tabular}

\section{CONCLUSIONES}

El caso que se ha utilizado para introducir el tema, no es algo que ocurrió hace años, es algo que sucede cotidianamente. La señora del ejemplo desarrolló cierta aversión hacia la actividad física. En vez de disfrutarla, ella asociará cualquier movimiento corporal con tortura. Tenemos que tener presente que cuando se trabaja con personas sedentarias se debe entender que el utilizar ejercicios apropiados para el mejoramiento de la resistencia abdominal, tiene un componente de prevención de problemas a nivel lumbar. Aunque en nuestro medio abundan los mitos y las malas prácticas al realizar actividad física, compete a los profesionales en las ciencias del movimiento educar a nuestra población en las prácticas adecuadas de la actividad física. Adicionalmente, tome en consideración que existen ejercicios abdominales que someten el área lumbar a una compresión muy alta de la zona, por lo que personas sedentarias deben recurrir al abdominal modificado. Sin embargo, el abdominal requerido para cada persona, especialmente con entrenamiento específico, debe considerar variables como: aptitud física, metas del entrenamiento, historia de lesiones en la espalda y cualquier otra referencia personal.

\section{BIBLIOGRAFÍA}

Alvarez, C. (1997). Manual de Fisiología del Ejercicio. UNA: Heredia, Costa Rica.

Craig, A. and McGill, S. (1997). Low back loads over a variety of abdominal exercises: searching for the safest abdominal challenge. Medicine and Science in Sports and Exercise. 12, 804811.

Juker, D., McGill and Kropf, P. (1996). Quantitative intramuscular myoelectric activity of quadratus lumborum during a wide variety of tasks. Clin. Biomech. 11, 170-172. 
Lamb, D. (1994). Physiology of Exercise. New York: MacMillan Publishing Company.

McGill, S. (1992). Myoelectrically based dynamic three-dimensional model to predict loads on lumbar spine tissues during lateral bending. J. Biomech. 25, 395-414.

Sallis, J. and Salens, B. (2000). Assessment of physical activity by self-report: status, limitations and future directions. RQSE. 71, 1-12.

Simons-Morton, B., Parcel, G. O’Hara, N., Blair, S., and Pate, R. (1988). Health-related physical fitness in childhood. Annual Review of Public Health. 9, 404-425.

Shepard, R., (1994). Physical activity and child health. Sport Medicine. 1, 205-233.

Trost, S., Pate, R., Dowda, M., Saunders, R., Ward, D., and Felton, G. (1996). Gender differences in physical activity and determinants of physical activity in rural fifth grade children. Journal of School Health. 66, 145-150.

Van Gender, N. and Marks, S. (1987). Aerobic Dance-Exercise Instructor Manual. California: IDAF Publisher.

Fecha de recepción del artículo: 04 de agosto del 2004.

Fecha de aceptación del artículo: 23 de setiembre del 2004.

Fecha de publicación del artículo: 24 de setiembre del 2004. 